\title{
Decreased levels of serum cytokeratin 19 fragment CYFRA 21-1 predict objective response to chemotherapy in patients with non-small cell lung cancer
}

\author{
LI PANG $^{1}$, JING WANG $^{2}$, YANWEN JIANG $^{2}$ and LIANGAN CHEN ${ }^{1}$ \\ ${ }^{1}$ Department of Respiratory Medicine, Chinese PLA General Hospital, Beijing 100853; \\ ${ }^{2}$ Department of Pulmonary and Critical Care Medicine, Beijing Shijitan Hospital, Beijing 100050, P.R. China
}

Received March 4, 2013; Accepted June 14, 2013

DOI: $10.3892 /$ etm.2013.1171

\begin{abstract}
Diagnostic tools capable of predicting early responses to chemotherapy are required to improve the individual management of cancer patients. The present study aimed to evaluate the prognostic significance of the serum tumor markers CYFRA 21-1, carcinoembryonic antigen (CEA), neuron-specific enolase (NSE), carbohydrate antigen (CA) 125, and CA 19-9 for predicting responses to different chemotherapy regimens in patients with non-small cell lung cancer (NSCLC). A total of 276 patients with postoperative stage I-IV NSCLC were retrospectively reviewed. The five tumor markers were measured before and after at least two cycles of chemotherapy using an electrochemiluminescent assay. Multivariate analysis revealed that performance status, age, postoperative stage and surgery were significantly associated with the response to chemotherapy. High baseline CYFRA 21-1 and CA 19-9 levels were associated with poor effectiveness of chemotherapy. Significant reductions in CYFRA 21-1 levels were associated with a positive response to various chemotherapy regimens. CEA, CA 125 and CA 19-9 expression was only associated with a positive response in patients receiving paclitaxel, docetaxel, pemetrexed and the epidermal growth factor receptor (EGFR) tyrosine kinase inhibitor (TKI). NSE expression was only associated with a positive response to gemcitabine. Receiver operating characteristic (ROC) curve analysis indicated that CYFRA 21-1 is the most sensitive of the tumor markers in predicting the response to chemotherapy. Serum CYFRA 21-1 is a useful surrogate marker for predicting the response to different chemotherapy regimens used to treat NSCLC and is a more sensitive marker than CEA, CA125, CA19-9 and NSE.
\end{abstract}

Correspondence to: Professor Liangan Chen, Department of Respiratory Medicine, Chinese PLA General Hospital, 28 Fuxing Road, Haidian, Beijing 100853, P.R. China

E-mail: chenliangan301@163.com

Key words: cytokeratin 19 fragment CYFRA 21-1, chemotherapy, non-small cell lung cancer, receiver operating characteristic analysis

\section{Introduction}

Lung cancer is one of the leading causes of morbidity and mortality worldwide (1). Non-small cell lung cancer (NSCLC) accounts for $>80 \%$ of total lung cancer cases. NSCLC may be removed surgically if diagnosed in the early stages (2). However, the majority of patients are diagnosed at more advanced stages of the disease when surgery is no longer possible. Such patients are candidates for chemotherapy, which is associated with high toxicity and high cost, with limited efficacy.

Patients undergoing chemotherapy require monitoring to assess tumor progression. Various techniques, including computed tomography (CT) and positron-emission tomography (PET), alone and in combination with specific biomarkers are routinely used (3). Compared with other methods, tumor markers provide an economical and convenient method for detecting early tumor recurrence $(4,5)$. Several markers have been shown to be sensitive and effective in monitoring NSCLC $(5,6)$. However, due to their lack of specificity, the identification of new markers for NSCLC progression in patients undergoing different chemotherapy regimens is required.

Cytokeratin 19 (CK 19) is a constituent of the intermediate filament protein responsible for the structural integrity of epithelial cells. It has been used as a surrogate biomarker of circulating tumor cells of epithelial origin, including adenocarcinoma. A fragment of CK 19, CYFRA 21-1, has been proposed as a tumor marker for several malignancies, including NSCLC $(5,7)$.

Carcinoembryonic antigen (CEA) is a cell surface anchoring protein. It is a member of the immunoglobulin cell adhesion molecule (IgCAM) superfamily and participates in cell adhesion. High serum concentrations of CEA have been shown to be associated with advanced disease and tumor relapse in patients with NSCLC and other cancers $(8,9)$.

The neuroendocrine marker neuron-specific enolase (NSE) is a glycolytic enzyme associated with high diagnostic sensitivity and specificity in small cell lung cancer (SCLC); however, its use in monitoring the effect of chemotherapy in NSCLC is controversial $(10,11)$.

The aim of the present study was to evaluate the prognostic significance of serum tumor markers in predicting the response to different chemotherapy regimens in patients with NSCLC. 


\section{Patients and methods}

Patients. Data from 276 patients diagnosed with NSCLC at the Department of Pulmonary and Critical Care Medicine, Beijing Shijitan Hospital, Capital Medical University (Beijing, China) between February 2009 and October 2012 were retrospectively reviewed. All patients had a recent histological diagnosis of NSCLC classified according to the World Health Organization criteria (12). None of the patients had received previous treatment for NSCLC and none had a history of other forms of malignancies. Clinical data were recorded, including age, gender, disease stage and Eastern Cooperative Oncologic Group (ECOG) performance status.

The majority of the patients (180 patients, $65.22 \%$ ) received a four-cycle chemotherapy regimen or 4 months of treatment with an epidermal growth factor receptor (EGFR) tyrosine kinase inhibitor (TKI). A number of patients (63 patients, $22.83 \%$ ) received no further treatment. However, 33 patients $(11.96 \%)$, who were in disease progression, only received two cycles of chemotherapy or 2 months of treatment with an EGFR kinase inhibitor.

Chemotherapy included third-generation platinum-based regimens used in combination with gemcitabine, paclitaxel, docetaxel or pemetrexed. Response, based on CT scans performed every 2 months after treatment, was evaluated according to the Response Evaluation Criteria in Solid Tumors (RECIST) criteria (13). Outcomes were classified as complete response (CR), partial response (PR), progressive disease (PD) or no change (NC). Written informed content was obtained from the patients. The study was approved by the Ethics Committee of Beijing Shijitan Hospital, Capital Medical University.

Blood samples. Blood samples were collected within 14 days before surgery or chemotherapy and 3 weeks after completion of the second or fourth chemotherapy cycles. On the two occasions, CYFRA 21-1, CEA, NSE, CA 125 and CA 19-9 were measured by automated electrochemiluminescent assays (Roche Diagnostics GmbH, Mannheim, Germany).

Statistical analysis. Statistical analysis was performed using SPSS version 16.0 (SPSS, Inc., Chicago, IL, USA). $\chi^{2}$ and logistic regression analyses were used to evaluate associations between tumor response and baseline clinical variables. Independent sample t-tests were used to analyze correlations between outcome and blood levels of CEA, CYFRA 21-1, CA 125, CA 19-9 and NSE. Numerical values for the tumor markers were transformed using natural logarithms to obtain near normal data distribution. Receiver operating characteristic (ROC) curves were created to evaluate the role of different tumor markers in predicting the response to chemotherapy. Cut-off values were determined by the Youden's index and the area under the curve was used as a measure of sensitivity. $\mathrm{P}<0.05$ was considered to indicate a statistically significant difference.

\section{Results}

Decline in CYFRA 21-1 predicts response for different chemotherapy in NSCLC. The baseline characteristics of the patients included in the study are shown in Table I. The study included
Table I. Baseline characteristics.

\begin{tabular}{lrr}
\hline Variable & $\mathrm{N}$ & $(\%)$ \\
\hline Gender & & \\
Male & 172 & 62.3 \\
Female & 104 & 37.7 \\
ECOG PS score & & \\
$\leq 1$ & 233 & 84.4 \\
$>1$ & 43 & 15.6 \\
Histological type & & \\
Squamous cell carcinoma & 72 & 26.1 \\
Other & 204 & 73.9 \\
Postoperative stage & & \\
I & 34 & 12.6 \\
II & 44 & 15.9 \\
IIIa & 27 & 9.8 \\
IIIb & 28 & 10.1 \\
IV & 143 & 51.8 \\
Surgery & & \\
Yes & 143 & 51.8 \\
No & 133 & 48.2 \\
First line treatment & & \\
NO & 63 & 22.8 \\
TP & 73 & 26.4 \\
GP & 54 & 19.6 \\
PP & 47 & 17.0 \\
TKI & 33 & 12.0 \\
Other & 6.2 \\
\hline & & \\
\hline
\end{tabular}

ECOG, Eastern Cooperative Oncology Group; PS, performance status; NO, surgery only; TP, paclitaxel or docetaxel; GP, gemcitabine; PP, pemetrexed; TKI, tyrosine kinase inhibitor.

172 males and 104 females with a median age of 63 years (range, 29-88 years). The majority of tumors were adenocarcinoma (70.7\%); squamous cell carcinoma (SCC) accounted for $26.1 \%$ of tumors and other histological types for $3.2 \%$. The majority of patients (51.8\%) received surgery, with the exception of those diagnosed as clinical Stage IV.

The treatment response was CR in 64 patients, PR in 47 patients, NC in 69 patients and PD in 96 patients. Based on the different treatment options, patients were divided into five groups as follows: a surgery only group (25 SCC and 38 adenocarcinoma patients); a paclitaxel or docetaxel (TP) group (19 SCC and 54 adenocarcinoma patients); a gemcitabine (GP) group (24 SCC and 30 adenocarcinoma patients); a pemetrexed (PP) group (0 SCC and 47 adenocarcinoma patients) and a TKI group (0 SCC and 33 adenocarcinoma patients). Thirty-two samples $(11.6 \%)$ were collected following the second cycle of treatment in cases where the patients were unable to tolerate further chemotherapy or their response was evaluated as PD.

Multivariate analysis revealed that performance status, age, postoperative stage and surgery were significantly correlated with the response to chemotherapy (Table II). However, there was no significant association between tumor response and 
Table II. Logistic regression analysis of the correlation between effectiveness of chemotherapy and clinical factors.

\begin{tabular}{lcccc}
\hline & & \multicolumn{2}{c}{$95 \%$ CI } & \\
\cline { 4 - 4 } Variable & Odds ratio & Lower & Upper & P-value \\
\hline ECOG PS score & 2.225 & 1.073 & 4.616 & $0.032^{\mathrm{a}}$ \\
Age & 1.053 & 1.009 & 1.099 & $0.018^{\mathrm{a}}$ \\
Gender & 0.759 & 0.353 & 1.631 & 0.480 \\
Histological type & 0.977 & 0.422 & 2.263 & 0.957 \\
Stage & 2.366 & 1.038 & 5.395 & $0.041^{\mathrm{a}}$ \\
Surgery & 3.441 & 1.431 & 8.273 & $0.006^{\mathrm{a}}$ \\
Chemotherapy regimen & 1.033 & 0.759 & 1.407 & 0.837 \\
CEA & 1.000 & 0.997 & 1.002 & 0.679 \\
CYFRA 21-1 & 1.010 & 0.983 & 1.038 & 0.465 \\
CA 125 & 0.999 & 0.997 & 1.002 & 0.327 \\
\hline
\end{tabular}

${ }^{\mathrm{a} P}<0.05$. ECOG, Eastern Cooperative Oncology Group; PS, performance status; CEA, carcinoembryonic antigen; CYFRA 21-1, cytokeratin 19 fragment; CA 125; carbohydrate antigen 125.

Table III. Correlation between effectiveness of chemotherapy and baseline serum levels of tumor markers.

\begin{tabular}{|c|c|c|c|c|c|}
\hline Variables & $\mathrm{N}$ & $\mathrm{CR}+\mathrm{PR}$ & $\mathrm{NC}+\mathrm{PD}$ & $\chi^{2}$ & P-value \\
\hline \multicolumn{6}{|c|}{ CYFRA 21-1 (ng/ml) } \\
\hline$<3.3$ & 98 & 53 & 45 & & \\
\hline$\geq 3.3$ & 130 & 50 & 80 & 3.935 & $0.047^{\mathrm{a}}$ \\
\hline \multicolumn{6}{|c|}{ CEA (ng/ml) } \\
\hline$<5.0$ & 143 & 63 & 80 & & \\
\hline$\geq 5.0$ & 129 & 47 & 82 & 1.636 & 0.201 \\
\hline \multicolumn{6}{|c|}{ CA $125(\mathrm{U} / \mathrm{ml})$} \\
\hline$<35$ & 135 & 69 & 66 & & \\
\hline$\geq 35$ & 107 & 34 & 73 & 0.710 & 0.791 \\
\hline \multicolumn{6}{|c|}{ CA 19-9 (U/ml) } \\
\hline$<37$ & 210 & 81 & 119 & & \\
\hline$\geq 37$ & 42 & 12 & 30 & 21.885 & $<0.001^{\mathrm{a}}$ \\
\hline \multicolumn{6}{|c|}{ NSE (ng/ml) } \\
\hline$<17$ & 145 & 70 & 75 & & \\
\hline$\geq 17$ & 68 & 25 & 43 & 2.482 & 0.115 \\
\hline
\end{tabular}

${ }^{a} \mathrm{P}<0.05$. CYFRA 21-1, cytokeratin 19 fragment; CEA, carcinoembryonic antigen; CA, carbohydrate antigen; NSE, neuron-specific enolase; $\mathrm{CR}$, complete response; $\mathrm{PR}$, partial response; $\mathrm{NC}$, no change; $\mathrm{PD}$, progressive disease.

gender, tumor type, chemotherapy regimen or baseline serum levels of tumor markers.

Patients were further divided into high and low tumor marker groups based on the pretreatment marker levels. The cut-off values that defined these groups are shown in Table III. Correlation analysis revealed that baseline CYFRA 21-1 ( $\mathrm{P}=0.047)$ and CA 19-9 $(\mathrm{P}<0.001)$ were significantly correlated with effectiveness of chemotherapy (Table III). No similar associations were identified for CEA, CA 19-9 and NSE (Table III).

Table IV shows the correlations between changes in tumor markers following chemotherapy and the effective- ness of different chemotherapy regimens. Reductions in the serum levels of CYFRA 21-1, CEA, CA 19-9 and CA 125 were significantly associated with outcome in the surgery only group. Reduced serum levels of CEA, CYFRA 21-1 and CA 19-9 were significantly correlated with effectiveness of TP and PP chemotherapy. CYFRA 21-1 and NSE were significantly associated with responses to gemcitabine. Responses in the TKI group were associated with reductions in the serum levels of CEA, CYFRA 21-1 and CA 125.

The reductions of tumor markers in relation to the effectiveness of chemotherapy are shown in Fig. 1 and Table V. Among all relevant markers, CYFRA 21-1 yielded the most 
Table IV. Correlation between responses of tumor markers and effectiveness of different chemotherapy regimens.

\begin{tabular}{|c|c|c|c|c|}
\hline Regimen & $\mathrm{CR}+\mathrm{PR}$ & $\mathrm{NC}+\mathrm{PD}$ & t-value & P-value \\
\hline \multicolumn{5}{|l|}{ NO } \\
\hline CEA & 25 & 36 & 3.953 & $<0.001^{\mathrm{a}}$ \\
\hline CYFRA 21-1 & 17 & 29 & 2.838 & $0.007^{\mathrm{a}}$ \\
\hline CA 19-9 & 21 & 25 & 2.178 & $0.039^{\mathrm{a}}$ \\
\hline CA 125 & 21 & 30 & 2.361 & $0.025^{\mathrm{a}}$ \\
\hline NSE & 19 & 22 & 1.303 & 0.200 \\
\hline \multicolumn{5}{|l|}{$\mathrm{TP}$} \\
\hline CEA & 34 & 39 & 4.889 & $<0.001^{\mathrm{a}}$ \\
\hline CYFRA 21-1 & 32 & 30 & 3.975 & $<0.001^{\mathrm{a}}$ \\
\hline CA $19-9$ & 24 & 26 & 2.290 & $0.026^{\mathrm{a}}$ \\
\hline CA 125 & 32 & 32 & 1.952 & 0.059 \\
\hline NSE & 23 & 21 & 1.571 & 0.124 \\
\hline \multicolumn{5}{|l|}{ GP } \\
\hline CEA & 25 & 27 & 1.912 & 0.062 \\
\hline CYFRA 21-1 & 22 & 20 & 2.419 & $0.020^{\mathrm{a}}$ \\
\hline CA $19-9$ & 21 & 22 & 1.004 & 0.327 \\
\hline CA 125 & 22 & 25 & 0.968 & 0.338 \\
\hline NSE & 20 & 18 & 3.003 & $0.004^{\mathrm{a}}$ \\
\hline \multicolumn{5}{|l|}{ PP } \\
\hline CEA & 21 & 26 & 3.813 & $<0.001^{\mathrm{a}}$ \\
\hline CYFR 21-1 & 20 & 17 & 2.715 & $0.010^{\mathrm{a}}$ \\
\hline CA 19-9 & 18 & 20 & 2.563 & $0.015^{\mathrm{a}}$ \\
\hline CA 125 & 10 & 19 & 1.201 & 0.238 \\
\hline NSE & 16 & 12 & 1.286 & 0.224 \\
\hline \multicolumn{5}{|l|}{ TKI } \\
\hline CEA & 5 & 22 & 4.696 & $<0.001^{\mathrm{a}}$ \\
\hline CYFRA 21-1 & 6 & 21 & 4.219 & $<0.001^{\mathrm{a}}$ \\
\hline CA 19-9 & 5 & 20 & 0.656 & 0.519 \\
\hline CA 125 & 5 & 19 & 2.424 & $0.024^{\mathrm{a}}$ \\
\hline NSE & 6 & 16 & 1.492 & 0.151 \\
\hline
\end{tabular}

${ }^{\mathrm{a}} \mathrm{P}<0.05$. NO, surgery only; TP, paclitaxel or docetaxel; GP, gemcitabine; PP, pemetrexed; TKI, tyrosine kinase inhibitor; CEA, carcinoembryonic antigen; CYFRA 21-1, cytokeratin 19 fragment; CA, carbohydrate antigen; NSE, neuron-specific enolase; CR, complete response; PR, partial response; $\mathrm{NC}$, no change; $\mathrm{PD}$, progressive disease.

promising sensitivity at $90 \%$. The corresponding area under the ROC curve was 0.779 (95\% CI, 0.700-0.858; $\mathrm{P}<0001)$.

\section{Discussion}

Chemotherapy is one of the main methods of treatment for NSCLC. Efficacy is routinely evaluated on the basis of radiological findings; however, this is not conducive to the early detection of recurrence and metastasis. Consequently, there is growing demand for convenient tools for estimating prognosis and for detecting responsiveness to therapy in order to optimize disease management on an individual basis. Several tumor markers, including CEA and CYFRA 21-1 in NSCLC, have been previously shown to provide useful estimates of prognosis (14-16). However, their role in the evaluation of treatment effectiveness is controversial, partly due to the lack of comparative studies evaluating different chemotherapy regimens.

It is well recognized that baseline parameters, including histology, gender, World Health Organization performance status, number of positive lymph nodes, gross tumor volume and high levels of tumor markers are associated with poor chemotherapy efficacy and poor survival $(5,6,17)$. In the present study we identified significant associations between performance status, age, postoperative stage and chemotherapy response; however, we did not identify similar associations for gender, tumor type, chemotherapy regimen or baseline tumor markers.

CYFRA 21-1 is a fragment of CK 19, mainly present in the cytoplasm of tumor cells of epithelial origin, including lung and esophageal cancer. Expression of CYFRA 21-1 has been detected in lung adenocarcinoma and SCC (15). 
Table V. Prognostic profile of biomarkers for responses to chemotherapy.

\begin{tabular}{lccccr}
\hline \multirow{2}{*}{ Marker } & AUC & $\begin{array}{c}\text { Sensitivity } \\
(\%)\end{array}$ & $\begin{array}{c}\text { Specificity } \\
(\%)\end{array}$ & Lower & Upper \\
\hline CEA & 0.738 & 81.4 & 55.6 & 0.654 & 0.822 \\
CYFRA 21-1 & 0.779 & 90.0 & 60.3 & 0.700 & 0.858 \\
CA 19-9 & 0.610 & 62.9 & 54.0 & 0.514 & 0.705 \\
CA 125 & 0.628 & 90.0 & 30.2 & 0.533 & 0.722 \\
NSE & 0.747 & 70.0 & 74.6 & 0.663 & 0.831 \\
\hline
\end{tabular}

AUC, area under the curve; CEA, carcinoembryonic antigen; CYFRA 21-1, cytokeratin 19 fragment; CA, carbohydrate antigen; NSE, neuron-specific enolase.

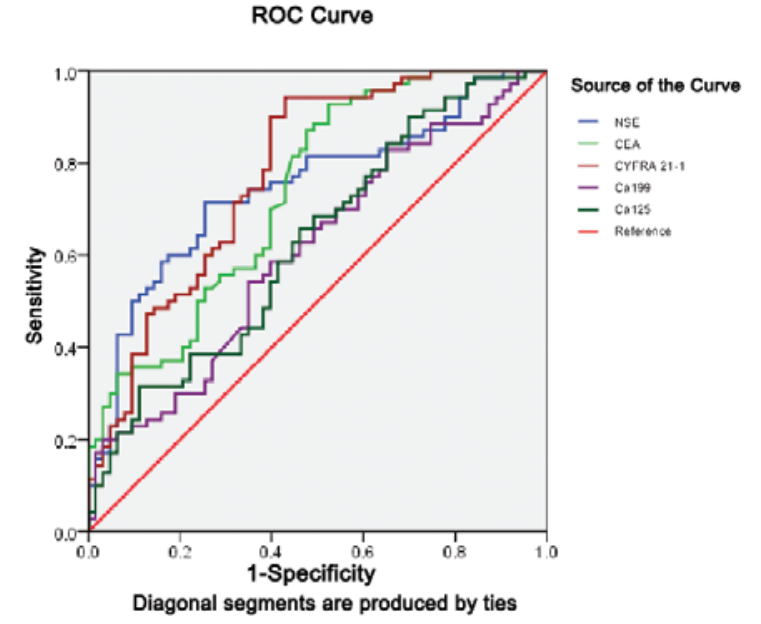

Figure 1. Receiver operating characteristic (ROC) curves for the responses of tumor markers in relation to the effectiveness of chemotherapy. NSE, neuronspecific enolase; CEA carcinoembryonic antigen; CYFRA 21-1, cytokeratin 19 fragment; CA, carbohydrate antigen.

CYFRA 21-1 has also been identified as a potential tumor marker for the diagnosis and prognosis of NSCLC. CYFRA 21-1 has previously been identified as a valuable marker for the individual management of patients with recurrent NSCLC receiving second-line chemotherapy (18). In the present study we demonstrated that high serum CYFRA 21-1 levels are indicative of chemotherapeutic effectiveness in NSCLC. This observation is consistent with a previous study reporting that baseline serum levels and changes in CYFRA 21-1 are reliable markers for chemotherapy response in NSCLC (15).

In the present study patients were analyzed in five groups according to the type of chemotherapy received. The majority of patients in the TP and GP groups had NSCLC, while all patients in the PP and TKI groups had adenocarcinoma. Comparison of the changes in serum tumor markers in the different groups revealed that reductions in CYFRA 21-1 were significantly associated with a positive response to chemotherapy in all groups. This finding may be related to the fact that CYFRA 21-1 expression is dependent on tumor stage as opposed to histological type (7,18-20). Two previous studies $(21,22)$ demonstrated that CYFRA $21-1$ is an indepen- dent prognostic factor in the earlier stages of SCC. However, data from another study (5) suggest that CYFRA 21-1 may be a reliable surrogate marker of chemotherapy efficacy in patients with advanced NSCLC.

CYFRA 21-1 is ubiquitously found in a number of body tissues and has been described as a marker of apoptotic cell death (23). This suggests that CYFRA 21-1 may be a tumor marker in SCLC as well as in NSCLC. One study demonstrated that high levels and insufficient reductions of CYFRA 21-1 during the first and second cycles of chemotherapy were correlated with poor outcome in 128 patients with newly diagnosed SCLC receiving first-line chemotherapy (19).

CEA is widely used as a tumor marker in lung, gastrointestinal and gynecological cancers, as well as other tumors. The majority of patients with NSCLC who have elevated CEA levels have adenocarcinoma (23-26). CEA is a member of the IgCAM superfamily and is involved in cell adhesion and transfer $(27,28)$. High serum levels of CEA have been shown to be a risk factor for the development of brain metastasis and are associated with poor prognosis (9). We identified no significant association between high baseline serum CEA levels and a poor response to chemotherapy. However, there was a significant association between decreased serum CEA levels and the effectiveness of chemotherapy in the groups where the majority of patients had adenocarcinoma. CEA may, therefore, represent a molecular target for lung adenocarcinoma. Similar results were observed for reductions in serum CA 125 and CA 19-9. All three markers are derived from glandular cells, which may make them suitable for monitoring the effectiveness of treatment in lung adenocarcinoma (29-31). However, these three markers are non-specific and it may be necessary to combine results for other biomarkers in order to increase sensitivity and specificity $(6,32,33)$.

Changes in serum NSE predicted responses only in the GP group. This may be explained by the fact that the majority of patients receiving this form of chemotherapy had SCC. Previous studies have identified NSE as a sensitive tumor marker for SCLC (10), but not for NSCLC $(20,34)$. More recent results are contradictory. One study concluded that patients without elevated NSE mRNA had a better prognosis compared with patients who experienced an increase in NSE, while another study concluded that pretreatment NSE mRNA had potential as a prognostic biomarker for advanced NSCLC $(20,35)$. 
Published evidence indicates that the most convincing data available among potential tumor markers for NSCLC, is that obtained with CYFRA 21-1 (15,18,36,37). In the present study, CYFRA 21-1 yielded the most promising sensitivity (90\%) and the largest area under the ROC curve, compared with the other tumor markers.

This retrospective investigation of prognostic tumor markers suggests that changes in serum levels of CYFRA 21-1 may be helpful in predicting the effectiveness of different chemotherapy regimens used to treat NSCLC. We also identified serum CEA, CA 125 and CA 19-9 as possible prognostic factors in lung adenocarcinoma.

\section{References}

1. Oken MM, Hocking WG, Kvale PA, et al: Screening by chest radiograph and lung cancer mortality: the Prostate, Lung, Colorectal, and Ovarian (PLCO) randomized trial. JAMA 306: 1865-1873, 2011

2. Ferlay J, Parkin DM and Steliarova-Foucher E: Estimates of cancer incidence and mortality in Europe in 2008. Eur J Cancer 46: 765-781, 2010.

3. Huang TW, Hsieh CM, Chang H, et al: Standard uptake value of positron emission tomography in clinical stage I lung cancer: clinical application and pathological correlation. Eur J Cardiothorac Surg 41: 869-873, 2012.

4. Sawabata N, Maeda H, Yokota S, et al: Postoperative serum carcinoembryonic antigen levels in patients with pathologic stage IA nonsmall cell lung carcinoma: subnormal levels as an indicator of favorable prognosis. Cancer 101: 803-809, 2004.

5. Ardizzoni A, Cafferata MA, Tiseo M, et al: Decline in serum carcinoembryonic antigen and cytokeratin 19 fragment during chemotherapy predicts objective response and survival in patients with advanced nonsmall cell lung cancer. Cancer 107: 2842-2849, 2006.

6. Dehing-Oberije C, Aerts H, Yu S, et al: Development and validation of a prognostic model using blood biomarker information for prediction of survival of non-small-cell lung cancer patients treated with combined chemotherapy and radiation or radiotherapy alone (NCT00181519, NCT00573040, and NCT00572325). Int J Radiat Oncol Biol Phys 81: 360-368, 2011.

7. Pujol JL, Molinier O, Ebert W, et al: CYFRA 21-1 is a prognostic determinant in non-small-cell lung cancer: results of a meta-analysis in 2063 patients. Br J Cancer 90: 2097-2105, 2004.

8. Grunnet M and Sorensen JB: Carcinoembryonic antigen (CEA) as tumor marker in lung cancer. Lung Cancer 76: 138-143, 2012.

9. Arrieta O, Saavedra-Perez D, Kuri R, et al: Brain metastasis development and poor survival associated with carcinoembryonic antigen (CEA) level in advanced non-small cell lung cancer: a prospective analysis. BMC Cancer 9: 119, 2009.

10. Emin Erbaycu A, Gunduz A, Batum O, Zeren Ucar Z, Tuksavul F and Zeki Guglu S: Pre-treatment and treatment-induced neuron-specific enolase in patients with small-cell lung cancer: an open prospective study. Arch Broncopneumol 46: 364-369, 2010 (In Spanish).

11. Jin B, Huang AM, Zhong RB and Han BH: The value of tumor markers in evaluating chemotherapy response and prognosis in Chinese patients with advanced non-small cell lung cancer. Chemotherapy 56: 417-423, 2010.

12. Travis WD: Pathology and genetics: tumours of the lung, pleura, thymus and heart. Volume 7. Iarc, 2004.

13. Eisenhauer EA, Therasse P, Bogaerts J, et al: New response evaluation criteria in solid tumours: revised RECIST guideline (version 1.1). Eur J Cancer 45: 228-247, 2009.

14. Seemann MD, Beinert T, Fürst $\mathrm{H}$ and Fink U: An evaluation of the tumour markers, carcinoembryonic antigen (CEA), cytokeratin marker (CYFRA 21-1) and neuron-specific enolase (NSE) in the differentiation of malignant from benign solitary pulmonary lesions. Lung Cancer 26: 149-155, 1999.

15. Edelman MJ, Hodgson L, Rosenblatt PY, et al: CYFRA 21-1 as a prognostic and predictive marker in advanced non-small-cell lung cancer in a prospective trial: CALGB 150304. J Thorac Oncol 7: 649-654, 2012.
16. Alatas F, Alatas O, Metintas M, Colak O, Harmanci E and Demir S: Diagnostic value of CEA, CA 15-3, CA 19-9, CYFRA 21-1, NSE and TSA assay in pleural effusions. Lung Cancer 31: $9-16,2001$

17. Li CG, Huang XE, Xu L, Li Y and Lu YY: Clinical application of serum tumor associated material (TAM) from non-small cell lung cancer patients. Asian Pac J Cancer Prev 13: 301-304, 2012.

18. Holdenrieder S, von Pawel J, Dankelmann E, et al: Nucleosomes and CYFRA 21-1 indicate tumor response after one cycle of chemotherapy in recurrent non-small cell lung cancer. Lung Cancer 63: 128-135, 2009.

19. Holdenrieder S, von Pawel J, Dankelmann E, et al: Nucleosomes, ProGRP, NSE, CYFRA 21-1, and CEA in monitoring first-line chemotherapy of small cell lung cancer. Clin Cancer Res 14: 7813-7821, 2008.

20. Alm El-Din MA, Farouk G, Nagy H, Abd Elzaher A and Abo El-Magd GH: Cytokeratin-19 fragments, nucleosomes and neuron-specific enolase as early measures of chemotherapy response in non-small cell lung cancer. Int J Biol Marker 27: e139-e146, 2012.

21. Kulpa J, Wójcik E, Reinfuss $M$ and Kolodziejski L: Carcinoembryonic antigen, squamous cell carcinoma antigen, CYFRA 21-1, and neuron-specific enolase in squamous cell lung cancer patients. Clin Chem 48: 1931-1937, 2002.

22. Song WA, Liu X, Tian XD, et al: Utility of squamous cell carcinoma antigen, carcinoembryonic antigen, Cyfra 21-1 and neuron specific enolase in lung cancer diagnosis: a prospective study from China. Chin Med J (Engl) 124: 3244-3248, 2011.

23. Sheard MA, Vojtesek B, Simickova M and Valik D: Release of cytokeratin-18 and -19 fragments (TPS and CYFRA 21-1) into the extracellular space during apoptosis. J Cell Biochem 85: 670-677, 2002.

24. Matsuoka K, Sumitomo S, Nakashima N, Nakajima D and Misaki N: Prognostic value of carcinoembryonic antigen and CYFRA21-1 in patients with pathological stage I non-small cell lung cancer. Eur J Cardiothorac Surg 32: 435-439, 2007.

25. Tomita M, Shimizu T, Ayabe T, Yonei A and Onitsuka T: Prognostic significance of tumour marker index based on preoperative CEA and CYFRA 21-1 in non-small cell lung cancer. Anticancer Res 30: 3099-3102, 2010.

26. Tomida M, Mikami I, Takeuchi S, Nishimura $\mathrm{H}$ and Akiyama H: Serum levels of nicotinamide N-methyltransferase in patients with lung cancer. J Cancer Res Clin Oncol 135: 1223-1229, 2009.

27. Iwanicki-Caron I, Di Fiore F, Roque I, et al: Usefulness of the serum carcinoembryonic antigen kinetic for chemotherapy monitoring in patients with unresectable metastasis of colorectal cancer. J Clin Oncol 26: 3681-3686, 2008.

28. Konstantopoulos K and Thomas SN: Cancer cells in transit: the vascular interactions of tumor cells. Annu Rev Biomed Eng 11: 177-202, 2009.

29. Thomas SN,Zhu F, Schnaar RL, Alves CS and Konstantopoulos K: Carcinoembryonic antigen and CD44 variant isoforms cooperate to mediate colon carcinoma cell adhesion to E- and L-selectin in shear flow. J Biol Chem 283: 15647-15655, 2008.

30. Thomas SN, Tong Z, Stebe KJ and Konstantopoulos K: Identification, characterization and utilization of tumor cell selectin ligands in the design of colon cancer diagnostics. Biorheology 46: 207-225, 2009.

31. Nuñez GR, Ito $C$ and Del Giglio A: Increased serum CA-125 levels in patients with lung cancer post thoracotomy. South Med J 102: 427-428, 2009.

32. Li X, Asmitananda T, Gao L, et al: Biomarkers in the lung cancer diagnosis: a clinical perspective. Neoplasma 59: 500-507, 2012.

33. Muley T, Fetz TH, Dienemann H, et al: Tumor volume and tumor marker index based on CYFRA 21-1 and CEA are strong prognostic factors in operated early stage NSCLC. Lung Cancer 60: 408-415, 2008

34. Ma S, Shen L, Qian N and Chen K: The prognostic values of CA125, CA19.9, NSE, AND SCC for stage I NSCLC are limited. Cancer Biomark 10: 155-162, 2011.

35. Wang Y, Tang D, Sui A, et al: Prognostic significance of NSE mRNA in advanced NSCLC treated with gefitinib. Clin Transl Oncol 15: 384-390, 2013.

36. Gridelli C, Rossi A and Maione P: Treatment of non-small-cell lung cancer: state of the art and development of new biologic agents. Oncogene 22: 6629-6638, 2003.

37. Wang J, Zhang N, Li B, et al: Decline of serum CYFRA21-1 during chemoradiotherapy of NSCLC: a probable predictive factor for tumor response. Tumour Biol 32: 689-695, 2011. 